\title{
Analysis of Short and Long Term Variability of Nasal Mucus versus Breath Condensate Inflammation Markers in Healthy Individuals
}

\section{Janssens Heleen ${ }^{1,2^{*}}$, De Prins Sofie ${ }^{1,2}$, Schoeters Greet ${ }^{1,2,3}$ and Koppen Gudrun ${ }^{1}$}

${ }^{1}$ Environmental Risk and Health Unit, Flemish Institute for Technological Research (VITO), Boeretang 200, B-2400 Mol, Belgium

${ }^{2}$ Faculty of Pharmaceutical, Biomedical and Veterinary Sciences, University of Antwerp, Belgium

${ }^{3}$ Institute of Public Health/Department of Environmental Medicine, University of Southern Denmark, Campusvej 55, DK-5230 Odense, Denmark

\begin{abstract}
Objective: Inflammation markers were measured in nasal mucus and exhaled breath condensate (EBC) to assess the normal variability of these markers among healthy individuals. The measurement of protein markers in those matrices is valuable to provide information on inflammation endotypes and local reaction of the immune system.
\end{abstract}

Methods: Inflammation markers (IFN- $y$, IL-1 1 , IL-8, IL-10, IL-13 and TNF- $\alpha$ ) in nasal mucus and EBC from six healthy adults were measured by Meso Scale Discovery technology. Nasal mucus was collected with sponges of polyurethane foam, which is a recently developed sampling technique. EBC was sampled via an optimized breath manoeuver with the RTUBETM device. Both collections were done within a limited sampling time of two minutes. The samples were taken on the same day and within a time period of twenty days for the determination of short and long term variability, respectively.

Results: All inflammation markers were detectable in nasal mucus. This was in contrast with the measurements in EBC, in which only IFN-y, IL-8 and IL-13 were detectable in the majority of the samples. EBC collected with the applied breathing technique gave a repeatable almost constant volume $(448 \mu \mathrm{L}(95 \% \mathrm{Cl}: 429$ to $467 \mu \mathrm{L}))$. The collected volume of nasal mucus was more variable (from 0 to $200 \mu \mathrm{L}$ ). The variability of nasal analytes was similar over the short and long time period. The nasal inflammation marker levels were more stable within an individual than between different individuals $(0.72 \leq \mathrm{ICC} \leq 0.96)$. This was in contrast with the measurements in $\mathrm{EBC}$ that were highly variable over time $(0.08 \leq \mathrm{ICC} \leq 0.51)$.

Conclusion: We indicate - given the good detectability and low variability of the inflammation markers determined in nasal mucus - that nasal mucus, more than EBC, is potentially a good matrix to assess inflammation in the respiratory system.

Keywords: Nasal mucus; Polyurethane foam; Exhaled breath condensate (EBC); RTube ${ }^{\mathrm{m}}$; Inflammation; Intra-class correlation coefficient (ICC)

\section{Introduction}

The measurements of protein markers in samples of the nasal, upper and lower airways are valuable to provide information on inflammation endotypes and local reaction of the immune system. The nose and bronchi show the same immune response to allergens causing inflammation of the airways [1-3]. During an inflammatory response, inflammatory cells like dendritic cells, macrophages, mast cells, basophils, eosinophils, neutrophils, $\mathrm{B}$ and $\mathrm{T}$ lymphocytes are activated and mediators such as leukotrienes, prostaglandins, histamine and cytokines are secreted [4-6]. These mediators are required for the communication between cells and they act on target cells to induce cellular functions such as activation, proliferation, growth, cell differentiation, apoptosis, chemotaxis and secretion of other mediators [7-9]. Cytokines can be divided into different groups with certain functions [10]: (a) pro-inflammatory cytokines that strengthen the inflammation, e.g. interleukin (IL)-1 $\beta$, tumor necrosis factor (TNF)- $\alpha$, IL-6, IL-11, granulocyte-macrophage colony-stimulating factor (GMCSF); (b) anti-inflammatory cytokines that weaken the inflammation, e.g. IL-10, interferon (IFN)- $\gamma$, IL-12, IL-18; (c) lymphokines that are predominantly secreted by $\mathrm{T}$ cells and regulate the immune response, e.g. IL-2, IL-3, IL-4, IL-5, IL-13, IL-15, IL-16, IL-17; (d) growth factors which promote the survival of cells and provide the structural changes in the airways, e.g. transforming growth factor (TGF)- $\beta$; and (e) chemokines with chemoattractant properties that help the recruitment of inflammatory cells to the site of inflammation, e.g. IL-8.
Various methods are available for sample collection of airway cell secretions. Invasive techniques such as bronchoalveolar lavages and biopsies are risky and not always well tolerated. There is a need for further exploration of non-invasive techniques such as sampling of nasal mucus and exhaled breath condensate (EBC) which can be applied to easily monitor large patient groups or study populations [11]. Nasal mucus is composed of $95 \%$ water, $2 \%$ mucin, $1 \%$ salts, $1 \%$ other proteins (such as albumin, immunoglobulins) and 1\% lipids [12]. It can be collected via lavage techniques, but sponges of e.g. polyurethane foam are useful for undiluted sampling [12-14]. In the current study we used sponges of polyurethane foam to collect nasal mucus on the epithelial surface. Exhaled breath consist of a gas phase and a vapor phase. EBC is obtained by cooling the vapor phase of the breath which contains aerosol particles. EBC is composed of $99 \%$ of water and $1 \%$ of nonvolatile compounds (such as cytokines, lipids, ions), and volatile water-soluble compounds (such as ammonia, hydrogen peroxide, ethanol) [15]. Research on EBC has been done since several years, but there is still room to improve the method of sampling [15-

*Corresponding author: Janssens Heleen, Flemish Institute for Technological Research (VITO), Boeretang 200, B-2400 Mol, Belgium Tel: +32 143351 56; Fax: +32 145805 23; E-mail: heleen.janssens@ vito.be

Received April 21, 2015; Accepted May 29, 2015; Published June 02, 2015

Citation: Janssens H, De Prins S, Schoeters G, Koppen G. (2015) Analysis of Short and Long Term Variability of Nasal Mucus versus Breath Condensate Inflammation Markers in Healthy Individuals. J Pulm Respir Med 5: 265. doi:10.4172/2161105X.1000265

Copyright: ( 2015 Heleen J, et al. This is an open-access article distributed under the terms of the Creative Commons Attribution License, which permits unrestricted use, distribution, and reproduction in any medium, provided the original author and source are credited. 
18]. We sampled EBC via an optimized breath manoeuver [19] with the portable collection device RTube ${ }^{\mathrm{rw}}[20]$. Using these state-of-theart methodologies the variability of inflammation markers within and between healthy individuals were determined to study their stability within one day and over a longer time period of twenty days.

\section{Methods}

\section{Study population and study design}

Six healthy adults 20-50 years old were recruited in April and May 2014. We did not set out any criterion for inclusion or exclusion of the participants in this study considering allergies and common colds. An even amount of men $(\mathrm{N}=3)$ and women $(\mathrm{N}=3)$ were included. All the subjects gave written informed consent to participate in the study. The study was approved by the ethical committee of the University of Antwerp (ID number: B300201316329).

This study had two parts: a short term and a long term follow-up. In the short term experiment nasal mucus and EBC were sampled from three subjects (three women) at six different time points on the same day between 8 a.m. and 6 p.m. In the long term experiment nasal mucus and EBC were sampled from six subjects on five different days in a twenty day period, always in the morning between 9 a.m. and 11 a.m. We did not restrict food intake by the participants in the time frame before the sampling, as this was not expected to influence inflammation marker levels.

\section{Sampling method}

Collection of nasal mucus: Sterilized sponges of polyurethane foam (Gummi Welz, Neu-Ulm, Germany) were used for the collection of nasal mucus. A sponge $(20 \times 15 \times 5 \mathrm{~mm})$ was placed in each nostril of the subject for two minutes. Both sponges were then placed in a 0.22 $\mu \mathrm{m}$ cellulose acetate Spin-X filter in a $2 \mathrm{~mL}$ centrifuge tube (Corning, USA). Centrifugation was done at $4500 \mathrm{rcf}$ for 10 minutes at $4^{\circ} \mathrm{C}$. The resulting nasal mucus was transferred with Maximum Recovery Tips (Axygen, Tewksbury, Massachusetts, USA) to a $1.5 \mathrm{~mL}$ Protein LoBind Eppendorf tube (Eppendorf, Hamburg, Germany). These special tips and tubes were used to avoid proteins from interacting with the plastic surface so loss of proteins would be minimal. The samples were stored at $-80^{\circ} \mathrm{C}$ until further analysis.

Collection of exhaled breath condensate: The portable sample device RTube ${ }^{\mathrm{m}}$ (Respiratory Research, Inc., Austin, Texas, USA) was used for the collection of EBC. Subjects were breathing through the RTube $^{\mathrm{mm}}$ for two minutes in a controlled way: in cycles of one second of inhalation and three seconds of deep exhalation according to the protocol of Johnson and Morawska [19]. No forced breathings (coughing, sneezing) were allowed. The exhaled air was cooled by means of an aluminum cylindric condenser that was cooled at $-20^{\circ} \mathrm{C}$ for at least 30 minutes prior to sampling. The resulting EBC was transferred with Maximum Recovery Tips to a $1.5 \mathrm{~mL}$ Protein LoBind Eppendorf tube. The samples were stored at $-80^{\circ} \mathrm{C}$ until further analysis.

\section{Inflammation markers analysis}

All samples of nasal mucus and EBC were analyzed with Meso Scale Discovery (MSD) Proinflammatory Panel 1 (human) kit (Meso Scale Discovery, Rockville, Maryland, USA). From this 10-spot multiplex kit six inflammation markers were measured: IFN- $\gamma$, IL$1 \beta$, IL-8, IL-10, IL-13 and TNF- $\alpha$. The MSD technology is based on multiplex sandwich enzyme-linked immunosorbent assay (ELISA) and electrochemiluminescent detection. From the collected nasal mucus $90 \mu \mathrm{L}$ was used for the analysis. In case there was not enough sample, nasal mucus was diluted with demineralized water. Thereafter $90 \mu \mathrm{L}$ of Diluent 2 of the MSD kit was added, resulting in at least 1/2 dilution. From the collected EBC $180 \mu \mathrm{L}$ was used for the analysis. Thereafter $90 \mu \mathrm{L}$ of Diluent 2 was added, resulting in $2 / 3$ dilution. The standards and nasal mucus samples were analysed in duplicate using $50 \mu \mathrm{L}$ per well. For the EBC samples three wells were used, each one containing of $75 \mu \mathrm{L}$. The rest of the procedure was performed according to the protocol of the MSD kit. The 96-well MSD plates were incubated while shaking at room temperature for three hours. After washing, $25 \mu \mathrm{L}$ of detection antibody solution was added to each well. The plates were further incubated while shaking at room temperature for two hours. After washing, $150 \mu \mathrm{L}$ of $2 \mathrm{X}$ Read Buffer $\mathrm{T}$ was added to each well. Afterwards, the plates were immediately read at $620 \mathrm{~nm}$ using a MESO QuickPlex SQ 120 (Meso Scale Discovery, Rockville, Maryland, USA). The standard curve range of the assay was $0.12-510 \mathrm{pg} / \mathrm{mL}$ for IL-8, IL$1 \beta$, and IL-13; $0.08-325 \mathrm{pg} / \mathrm{mL}$ for IL-10 and TNF- $\alpha ; 0.3-1230 \mathrm{pg} / \mathrm{mL}$ for IFN $-\gamma$. The theoretical detection limits of respectively IFN- $\gamma$, IL- $1 \beta$, IL-8, IL-10, IL-13 and TNF- $\alpha$ were $0.20,0.04,0.04,0.03,0.24$ and 0.04 $\mathrm{pg} / \mathrm{mL}$. Samples below the detection limit were given half of the value of the lowest calculated concentration.

\section{Data analysis}

Descriptive values such as mean, standard error, minimum, lower quartile $\left(\mathrm{P}_{25}\right)$, median $\left(\mathrm{P}_{50}\right)$, upper quartile $\left(\mathrm{P}_{75}\right)$ and maximum were calculated for all measurements in EBC and nasal mucus. The normality of the inflammation marker levels was checked using the ShapiroWilk's $\mathrm{W}$ test. The natural logarithm was taken to obtain a normal distribution of the data. A repeated measures analysis of variance (ANOVA) was performed with STATISTICA version 10 (StatSoft, Inc., Tulsa, Oklahoma, USA) to calculate the intra-class correlation coefficient (ICC) of the ratio of between-individual variance divided by the sum of between-individual and within-individual variance. The ICC gives an indication of the variability of the repeated measurements within one individual. ICC values were interpreted as following: $<0.40$ weak agreement, 0.40 - 0.75 fair to good agreement and $>0.75$ excellent agreement $[2,21]$.

\section{Results}

\section{Collected volume of nasal mucus and EBC}

In total $45 \mathrm{EBC}$ and 42 nasal mucus samples were collected from six healthy individuals. The sampling methods were well tolerated by all participants.

The volume of nasal mucus collected for two minutes with nasal sponges varied greatly, with yields between 0 and $200 \mu \mathrm{L}$. Three collections resulted in no nasal fluid sample. The coefficient of variation for all the collected volumes of nasal mucus for the short time experiment was $44.91 \%$. For the long term experiment this was $97.11 \%$. When the collected volume of nasal mucus was too low for the analysis, the sample was diluted with demineralized water.

A relatively constant volume of EBC was obtained with the RTube ${ }^{\mathrm{rw}}$, independently of the individual being sampled. After two minutes of breathing in a controlled way (in cycles of one second of inhalation and three seconds of deep exhalation according to the protocol of Johnson and Morawska [19]) in the RTube ${ }^{\mathrm{ma}}$, the mean collected volume of EBC was $448 \mu \mathrm{L}$ (95\% confidence interval (CI): 429 to $467 \mu \mathrm{L}$ ). The coefficient of variation for all the collected volumes of $\mathrm{EBC}$ for the short time experiment was $15.21 \%$. For the long term experiment this was $13.53 \%$. 
Citation: Janssens H, De Prins S, Schoeters G, Koppen G (2015) Analysis of Short and Long Term Variability of Nasal Mucus versus Breath Condensate Inflammation Markers in Healthy Individuals. J Pulm Respir Med 5: 265. doi:10.4172/2161-105X.1000265

\section{Descriptive values of inflammation markers in EBC and nasal mucus}

The concentrations of the inflammation markers in EBC were very low and many were below the detection limit. IFN- $\gamma$, IL-8 and IL-13 were detectable in the majority of samples (Table 1). In nasal mucus all inflammation markers could be easily detected in undiluted as well as diluted samples (Table 2). The concentration of nasal IL-8 was not quantifiable due to concentrations above the highest standard $(>529.1$ $\mathrm{pg} / \mathrm{mL}$ ).

\section{Short term variability}

All samples of nasal mucus and EBC were collected for all three female volunteers. The variability of repeated measurements of nasal mucus on the same day was low, in contrast to the measurements in EBC that were highly variable. The ICCs of nasal mucus inflammation marker measurements were all excellent (Table 3), which means that the agreement of measurements within a person was higher than between the individuals. The ICC of EBC inflammation marker measurements were weak (Table 3), only IL-8 levels in EBC were more stable. ANOVA couldn't be performed for measurements of IL-1 $\beta$ and IL-10 in EBC and IL-8 in nasal mucus, because concentrations were respectively below or above the standard curve range.

\section{Long term variability}

EBC was collected in all individuals. Three samples of nasal mucus were missing: two for person 6 (male) on the third and fifth day and one for person 5 (male) on the fifth day. The variability of repeated measurements of nasal mucus for the twenty day period was low, in contrast to the measurements in EBC that were highly variable. The ICC of nasal mucus inflammation marker measurements were all excellent (Table 3), which means that the agreement of measurements within a person was higher than between the individuals. The ICC of EBC inflammation marker measurements were weak (Table 3), only TNF- $\alpha$ levels in EBC were more stable. ANOVA couldn't be performed for measurements of IL- $1 \beta$ in EBC and IL- 8 in nasal mucus, because concentrations were respectively below or above the standard curve range.

The ICC values of short and long term nasal analyte levels were similar (Table 3), except for IFN- $\gamma$ that had a better stability on the same day than on the long term. On the opposite, IL-13 had a better stability on the long term compared to the within-one-day follow-up.

\section{Discussion}

Measuring inflammation markers in nasal mucus and EBC can provide valuable information about inflammation endotypes and local reaction of the immune system [11,22]. Due to a strong functional and immunological relationship between the nose and the bronchi, the inflammatory response in the nose is similar to the situation in the lungs [1-3].

Nasal mucus can be collected in order to measure inflammation markers in the nasal airways [11]. The nose is one of the first places of contact with the outer environment and is therefore an interesting tissue. The nasal mucosa doesn't only come in contact with pollen, but

\begin{tabular}{|c|c|c|c|c|c|c|}
\hline Analyte & Mean $( \pm$ SE) & Min & P25 & P50 & P75 & Max \\
\hline IFN- - & $0.0451( \pm 0.0090)$ & 0.0011 & 0.0011 & 0.0178 & 0.0764 & 0.2624 \\
\hline IL-1 $\beta$ & $0.0033( \pm 0.0012)$ & 0.0008 & 0.0008 & 0.0014 & 0.0014 & 0.0446 \\
\hline IL-8 & $0.0244( \pm 0.0032)$ & 0.0026 & 0.0056 & 0.0204 & 0.0329 & 0.0865 \\
\hline IL-10 & $0.0036( \pm 0.0009)$ & 0.0002 & 0.0002 & 0.0019 & 0.0045 & 0.0373 \\
\hline IL-13 & $1.1944( \pm 0.0947)$ & 0.1715 & 0.7667 & 0.9803 & 1.8007 & 2.4779 \\
\hline TNF- $\alpha$ & $0.0075( \pm 0.0012)$ & 0.0002 & 0.0002 & 0.0063 & 0.0114 & 0.0275 \\
\hline
\end{tabular}

Table 1: Descriptive values for all 45 collected EBC samples: mean \pm standard error (SE), minimum (Min), lower quartile (P25), median (P50), upper quartile (P75) and maximum (Max) in $\mathrm{pg} / \mathrm{mL}$ per analyte.

\begin{tabular}{|c|c|c|c|c|c|c|}
\hline Analyte & Mean ( \pm SE) & Min & P25 & P50 & P75 & Max \\
\hline IFN-Y & $207.6( \pm 130.1)$ & 0.4 & 2.0 & 14.1 & 32.2 & 5250.5 \\
\hline IL-1 $\beta$ & $155.4( \pm 64.1)$ & 3.7 & 15.5 & 40.1 & 105.5 & 2521.9 \\
\hline IL-8 & $>529.1$ & $>529.1$ & $>529.1$ & $>529.1$ & $>529.1$ & $>529.1$ \\
\hline IL-10 & $6.6( \pm 1.3)$ & 0.3 & 1.3 & 3.3 & 7.9 & 39.8 \\
\hline IL-13 & $43.9( \pm 7.5)$ & 1.5 & 18.0 & 28.6 & 52.2 & 291.6 \\
\hline TNF- $\alpha$ & $12.1( \pm 1.8)$ & 0.5 & 3.0 & 7.7 & 18.8 & 51.9 \\
\hline
\end{tabular}

Table 2: Descriptive values for all 42 collected nasal mucus samples: mean \pm standard error (SE), minimum (Min), lower quartile (P25), median (P50), upper quartile (P75) and maximum (Max) in $\mathrm{pg} / \mathrm{mL}$ per analyte.The theoretical detection limit of respectively IFN- $\mathrm{y}, \mathrm{IL}-1 \beta, \mathrm{IL}-8, \mathrm{IL}-10, \mathrm{IL}-13$ and TNF- $\alpha$ were $0.20,0.04,0.04,0.03,0.24$ and $0.04 \mathrm{pg} / \mathrm{mL}$.

\begin{tabular}{|c|c|c|c|c|}
\hline \multirow{2}{*}{ Analyte } & \multicolumn{2}{|c|}{ Short term variability } & \multicolumn{2}{c|}{ Long term variability } \\
\cline { 2 - 4 } & ICC nasal mucus & ICC EBC & ICC nasal mucus & 0.72 \\
\hline IFN-Y & 0.96 & 0.08 & 0.90 \\
\hline IL-1 $\beta$ & 0.96 & $/$ & $/$ & 0.26 \\
\hline IL-8 & $/$ & 0.44 & 0.84 \\
\hline IL-10 & 0.87 & $/$ & 0.87 & 0.23 \\
\hline IL-13 & 0.77 & 0.32 & 0.89 \\
\hline TNF- $\alpha$ & 0.93 & 0.23 & 0.33 \\
\hline
\end{tabular}

Table 3: The calculated intra-class correlation coefficient (ICC) of the measurements in nasal mucus and EBC per analyte for the short and long term follow-up. /: below or above standard curve range 
also with bacteria, viruses, particles, air pollutants and irritants [2]. Sponges of polyurethane foam are recently used as collectors for nasal mucus. Riechelmann et al. [13] showed how the use of these sponges was superior to other tested techniques (nasal lavage, nasal spray blow technique and filter paper) for recovery of inflammation markers [13]. In the current study inflammation markers (IFN- $\gamma$, IL-1 $\beta$, IL-8, IL10 , IL-13 and TNF- $\alpha$ ) were measured in nasal mucus collected with this type of sponges. This sampling method was well tolerated by all participants. However, placement of a foreign substance for a period of time may traumatize the mucosa and alter concentrations of analytes under investigation $[13,14]$. In our study we repeated the sampling every two hours during one day and we didn't observe any increase in analyte levels. Because of the short contact time of two minutes and the inert nature of the material used, there was little chance of traumatization of tissue or irritation. The concentrations of nasal analytes were all easily detected and all within or above the range of the standard curve. Good detectable levels of markers in nasal mucus are certainly an advantage compared to those in EBC. Nasal secretions can be collected without dilution, whereas the inflammation marker levels in EBC are 10000 times lower than the concentrations in lung lining fluid $[11,17,18]$. The measured concentrations of nasal inflammation markers in the current study were of the same order or higher than those reported in other studies $[1,13,14,23]$. The concentrations of the nasal inflammation markers were approximately 1000 times higher than the concentrations in EBC. McDougall et al. [24] and Pringle et al. [25] reported that some nasal epithelial cells' mediators such as IL- 8 and IL-6 had an increased constitutive expression compared to bronchial epithelial cells $[24,25]$.

A disadvantage of the nasal mucus collector was the highly variable collected volume per person and per sample time point. This is possibly related to the inter-individual differences in morphology and accessibility of the nasal mucosa at the height of the nasal septum and the placement of the collector. The volume of collected nasal mucus after two minutes varied between 0 to $200 \mu \mathrm{L}$. If too little volume is collected, the nasal fluid can be diluted with a known factor [1,1214]. We have applied this method for some samples, but we didn't test the influence of ex vivo nasal mucus dilution on the measured concentration of inflammation markers. Alternatively, more sample of nasal mucus could be pooled from repeated samplings on the same day since we showed a low variability within an individual. However this methodology was not tested in this study.

EBC can be collected in order to measure inflammation markers in the lungs, although there is some discussion on its reproducible collection. The sampling of EBC is simple, non-invasive and safe [1518]. When breathing normal for 5 to 15 minutes, approximately 1 $\mathrm{mL}$ of EBC can be collected [15]. In the current study an optimized breathing technique was tested, which is based on the mechanism of breath aerosol formation by Johnson and Morawska [19]. They showed that the aerosol production was higher in case of a fast inhalation and deep exhalation [19]. In the current study the participants had to breath for two minutes in the RTube ${ }^{\mathrm{m}}$ in a controlled way, i.e. in cycles of one second of inhalation and three seconds of deep exhalation. This optimized breathing technique would be more difficult to apply with young children, who do not have the needed breathing coordination skills. However, in the current study with adults, a sufficient and fairly constant volume of EBC ( $448 \mu \mathrm{L}$ (95\% CI: 429 to $467 \mu \mathrm{L})$ ) could be collected for analysis in a very short time. Since the RTube ${ }^{\mathrm{mu}}$ has the disadvantage that the condensation temperature can vary during sampling [16], shortening the sampling time was supposed to increase the stability of the EBC analyte levels. However, the concentrations of the inflammation markers (IFN- $\gamma$, IL- $1 \beta$, IL-8, IL-10, IL-13 and TNF- $\alpha$ ) were low and often below the detection limit. On the other hand, as IFN- $\gamma$, IL- 8 and IL- 13 were detected, EBC could be an appropriate matrix for some inflammation markers. The measured concentrations of EBC inflammation markers were lower than or equal to other reported literature values. Different collectors, analysis methods and/ or sample processing procedures could explain these differences $[9,26-$ 28 ]. IL-1 $\beta$ in EBC was of the same order of previous measurements in children in which EBC was sampled using the RTube ${ }^{\mathrm{Tw}}$ and analysed in our lab [20].

The measurements in EBC were highly variable over time $(0.08$ $\leq$ ICC $\leq 0.51$ ), except for the relative stability of IL- 8 during one day $(\mathrm{ICC}=0.44)$ and TNF- $\alpha$ over a twenty day period $(\mathrm{ICC}=0.51)$. The higher variation of analytes in EBC was possibly due to a higher technical variability of measuring in the lower range of the MSD method.

The variability of nasal analytes was similar over the short and long time period. The nasal inflammation marker levels were more stable within an individual than between different individuals $(0.72 \leq$ ICC $\leq$ 0.96). IFN- $\gamma$ had a better stability within the same day than within the long term period. IFN- $\gamma$ is a Th 1 cytokine and is known to be expressed in e.g. common colds $[4,7,26]$. Therefore it can be expected that IFN- $\gamma$ is more variable over a longer period. Unexpectedly, IL-13 had a better stability in the long term follow-up than on the same day. IL-13 is a Th2 cytokine which plays a role in isotope switching to Immunoglobulin $\mathrm{E}$ (IgE) production and is important for sensitisation $[4,6,7,26,29]$. The correlation between inflammation markers was tested with Spearman rank correlation. The nasal levels of inflammation markers were all correlated (see Supplementary information: Table S.I), except for IFN- $\gamma$ (Th1 cytokine) and IL-13 (Th2 cytokine) indicative for their different role in inflammation. We didn't observe a correlation between inflammation markers in nasal mucus and EBC. We speculate that this is due to the low detectability of inflammation markers in EBC.

Some limitations of this study were the limited number of participants, and the loss of statistical power because of non-results due to dry sponges. We also did not investigate the relationship between short and long term information on disease/infection, allergen exposure or pollutants and the respective fluctuation of inflammation markers. Certainly, reference and relevant clinical values need to be established based on larger study populations performing allergen challenges and longitudinal sampling post exposure. A clinical relevant increase of a factor 10 for e.g. IL-13 and INF- $\gamma$ was observed by Scadding et al. [14], who performed a nasal challenge with purified Timothy grass allergen. These high levels were not seen in the current study population.

\section{Conclusion}

Undiluted nasal mucus was collected in a simple way using polyurethane sponges. In case the sampled mucus volume was too low, a larger amount could be obtained by repeated sampling on the same day because of the low variability of nasal inflammation markers within an individual. The fact that the nose is a first contact tissue of e.g. allergens, combined with nasal inflammation markers being more stable over time and easily detectable, makes nasal secretion - more than breath condensate - a potentially good matrix to assess inflammation in the respiratory tract.

\section{Acknowledgments}

Thanks to Dr. Guy W. Scadding from Imperial College Londen (UK) for kindly supplying us with polyurethane sponges and practical information on how to sample and handle the nasal mucosa samples. 
Citation: Janssens H, De Prins S, Schoeters G, Koppen G (2015) Analysis of Short and Long Term Variability of Nasal Mucus versus Breath Condensate Inflammation Markers in Healthy Individuals. J Pulm Respir Med 5: 265. doi:10.4172/2161-105X.1000265

\section{References}

1. Chawes BL, Edwards MJ, Shamji B, Walker C, Nicholson GC, et al. (2010) A novel method for assessing unchallenged levels of mediators in nasal epithelial lining fluid. J Allergy Clin Immunol 125: 1387-1389.

2. AIAhmari MD, Sapsford RJ, Wedzicha JA, Hurst JR (2011) Intersession repeatability of a novel nasal lavage technique. Transl Res 158: 163-168.

3. Raulf-Heimsoth M, Wirtz C, Papenfuss F, Baur X (2000) Nasal lavage mediator profile and cellular composition of nasal brushing material during latex challenge tests. Clin Exp Allergy. 30(1): 110-121.

4. Agrawal DK, Shao Z (2010) Pathogenesis of allergic airway inflammation. Curr Allergy Asthma Rep 10: 39-48.

5. Galli SJ, Tsai M, Piliponsky AM (2008) The development of allergic inflammation. Nature 454: 445-454.

6. Murdoch JR, Lloyd CM (2010) Chronic inflammation and asthma. Mutat Res 690: 24-39.

7. Barnes PJ, Chung KF, Page CP (1998) Inflammatory mediators of asthma: an update. Pharmacol Rev 50: 515-596.

8. Barnes PJ (2008) The cytokine network in asthma and chronic obstructive pulmonary disease. J Clin Invest 118: 3546-3556.

9. Robroeks CM, van de Kant KD, Jöbsis Q, Hendriks HJ, van Gent R, et al. (2007) Exhaled nitric oxide and biomarkers in exhaled breath condensate indicate the presence, severity and control of childhood asthma. Clin Exp Allergy 37: 1303-1311.

10. Chung KF, Barnes PJ (1999) Cytokines in asthma. Thorax 54: 825-857.

11. Alexis NE (2014) Biomarker sampling of the airways in asthma. Curr Opin Pulm Med 20: 46-52.

12. Lü FX, Esch RE (2010) Novel nasal secretion collection method for the analysis of allergen specific antibodies and inflammatory biomarkers. J Immunol Methods 356: 6-17.

13. Riechelmann H, Deutschle T, Friemel E, Gross HJ, Bachem M (2003) Biologica markers in nasal secretions. Eur Respir J 21: 600-605.

14. Scadding GW, Calderon MA, Bellido V, Koed GK, Nielsen NC, et al. (2012) Optimisation of grass pollen nasal allergen challenge for assessment of clinical and immunological outcomes. J Immunol Methods 384: 25-32.

15. Hunt $J$ (2002) Exhaled breath condensate: an evolving tool for noninvasive evaluation of lung disease. J Allergy Clin Immunol 110: 28-34

16. Kuban P, Foret $F(2013)$ Exhaled breath condensate: determination of nonvolatile compounds and their potential for clinical diagnosis and monitoring. $A$ review. Anal Chim Acta 805: 1-18.

17. Montuschi P, Barnes PJ (2002) Analysis of exhaled breath condensate for monitoring airway inflammation. Trends Pharmacol Sci 23: 232-237.

18. Montuschi P (2007) Analysis of exhaled breath condensate in respiratory medicine: methodological aspects and potential clinical applications. Ther Adv Respir Dis 1: 5-23.

19. Johnson GR, Morawska L (2009) The mechanism of breath aerosol formation. J Aerosol Med Pulm Drug Deliv 22: 229-237.

20. De Prins S, Dons E, Van Poppel M, Int Panis L, Van de Mieroop E, et al. (2014) Airway oxidative stress and inflammation markers in exhaled breath from children are linked with exposure to black carbon. Environ Int 73: 440-446.

21. Wielgomas B (2013) Variability of urinary excretion of pyrethroid metabolites in seven persons over seven consecutive days--implications for observational studies. Toxicol Lett 221: 15-22.

22. Puntmann VO (2009) How-to guide on biomarkers: biomarker definitions, validation and applications with examples from cardiovascular disease. Postgrad Med J 85: 538-545.

23. Følsgaard NV, Chawes BL, Rasmussen MA, Bischoff AL, Carson CG, et al (2012) Neonatal cytokine profile in the airway mucosal lining fluid is skewed by maternal atopy. Am J Respir Crit Care Med 185: 275-280.

24. McDougall CM, Blaylock MG, Douglas JG, Brooker RJ, Helms PJ, et al. (2008) Nasal epithelial cells as surrogates for bronchial epithelial cells in airway inflammation studies. Am J Respir Cell Mol Biol 39: 560-568.

25. Pringle EJ, Richardson HB, Miller D, Cornish DS, Devereux GS, et al. (2012 Nasal and bronchial airway epithelial cell mediator release in children. Pediatr Pulmonol 47: 1215-1225.

26. Robroeks CM, Rijkers GT, Jöbsis Q, Hendriks HJ, Damoiseaux JG, et al. (2010) Increased cytokines, chemokines and soluble adhesion molecules in exhaled breath condensate of asthmatic children. Clin Exp Allergy 40: 77-84.

27. Matsunaga K, Yanagisawa S, Ichikawa T, Ueshima K, Akamatsu K, et al (2006) Airway cytokine expression measured by means of protein array in exhaled breath condensate: correlation with physiologic properties in asthmatic patients. J Allergy Clin Immunol 118: 84-90.

28. Rosias PP, Robroeks CM, Kester A, den Hartog GJ, Wodzig WK, et al. (2008) Biomarker reproducibility in exhaled breath condensate collected with different condensers. Eur Respir J 31: 934-942.

29. Bloemen K, Verstraelen S, Van Den Heuvel R, Witters H, Nelissen I, et al. (2007) The allergic cascade: review of the most important molecules in the asthmatic lung. Immunol Lett 113: 6-18. 